\title{
Use of pars plana vitrectomy with phacoemulsification in vitreous cavity to treat complete posterior dislocation of lens
}

This article was published in the following Dove Press journal:

Clinical Ophthalmology

6 July 20II

Number of times this article has been viewed

\section{Tetsuya Mutoh \\ Yukihiro Matsumoto \\ Makoto Chikuda \\ Department of Ophthalmology, Dokkyo Medical University Koshigaya Hospital, Koshigaya, Saitama, Japan}

Background: The purpose of this study was to evaluate pars plana vitrectomy (PPV) with phacoemulsification in the vitreous cavity for treatment of complete posterior dislocation of the lens without any damage to the lens capsule.

Methods: We evaluated factors such as cause of dislocation, scleral fixation of an intraocular lens (IOL), preoperative and postoperative visual acuity, corneal endothelial cell density, and intraoperative and postoperative complications.

Results: Displacement in the eleven eyes studied was mostly caused by ocular trauma and was idiopathic (four eyes each). Scleral fixation of the IOL was performed in seven of the eyes during first-time PPV. Visual acuity was improved in two eyes, unchanged in eight eyes, and worse in one eye. Mean corneal endothelial cell density was 2485 cells $/ \mathrm{mm}^{2}$ preoperatively and 2301 cells $/ \mathrm{mm}^{2}$ postoperatively. No significant differences were seen before and after the surgeries $(P=0.15$, paired $t$-test). Intraoperative complications included retinal detachment and suprachoroidal hemorrhage in one eye each. The most common postoperative complication was transient ocular hypertension, which occurred in three eyes. IOL dislocation and vitreous incarceration of the anterior chamber in one eye required a second operation, while retinal detachment in one eye required a third operation.

Conclusion: When treating a completely dislocated lens, prevention of retinal detachment which subsequently can affect the visual prognosis is the most important factor to be addressed after PPV with intravitreal phacoemulsification.

Keywords: lens dislocation, pars plana vitrectomy, phacoemulsification in the vitreous cavity, retinal detachment

\section{Introduction}

Extraction of a completely dislocated lens with an intact capsule from the vitreous cavity has long been a difficult surgical problem. ${ }^{1}$ When the dislocated lens is retained in the vitreous cavity, this can lead to poor visual acuity, along with inflammation, corneal edema, and glaucoma. ${ }^{2}$

Development of techniques and instruments in the vitreoretinal surgery field has led to improved surgical results along with a decreased number of complications. As a result, more active surgical treatments are now being undertaken in some vitreoretinal diseases, such as treatments for a completely dislocated lens with an intact capsule in the vitreous cavity. This is very different from the very conservative approaches that would have been attempted in such cases in the past. ${ }^{3}$ Pars plana vitrectomy (PPV) with lensectomy techniques are well suited to cases in which there is complete dislocation of the lens with an intact capsule. ${ }^{4}$
Department of Ophthalmology, Dokkyo Medical University Koshigaya Hospital, Koshigaya, Saitama 343-8555, Japan

Tel +8I 48965 IIII

Fax +8I 48965 II 27

Email onihei@bu.edu 
However, despite current advances, manipulation of the lens intravitreally still involves a potentially high risk of retinal injury. ${ }^{1}$ The use of perfluorocarbon liquid has made PPV safer, because it allows the lens to float over the retinal surface, thereby avoiding retinal injury. ${ }^{5}$ In this study, we retrospectively evaluated outcomes in patients with a completely dislocated lens who underwent PPV and intravitreal phacoemulsification using perfluorocarbon liquid.

\section{Methods and materials}

We reviewed the medical records of eleven patients (eleven eyes, nine men, two women) who underwent PPV between September 2006 and November 2010 as treatment for a completely dislocated lens with an intact anterior and posterior capsule. All surgeries were performed by one experienced surgeon at the Dokkyo Medical University Koshigaya Hospital. All patients were followed for a minimum of 3 months (mean follow-up $13.1 \pm 13.5$ months). The mean age of the patients was $64.1 \pm 9.5$ years.

A standard 20-gauge three-port PPV was performed by removing enough vitreous and posterior hyaloid membrane to free the dislocated lens. After $0.1-0.3 \mathrm{cc}$ of perfluorocarbon liquid was injected at the posterior pole, the lens was lifted and subsequently positioned in the vitreous cavity. Phacoemulsification was performed, with great care taken to ensure the ultrasound energy was in the middle of the vitreous cavity. Small lens flakes attached to the retina were removed using a silicone tip connected to the flute needle.

In cases where scleral fixation of the intraocular lens (IOL) was performed, a foldable acrylic IOL (VA-65BB or YA-65BB, Hoya Corporation, Tokyo, Japan) was used. The surgical method for scleral fixation of the IOL has been reported previously. ${ }^{6}$ Briefly, after a $3.5 \mathrm{~mm}$ superior scleral tunnel incision was made, triangular scleral flaps were created opposite to each other at the $8 \mathrm{o}^{\prime}$ clock and $2 \mathrm{o}^{\prime}$ clock positions, with the IOL then sutured using an ab externo approach. ${ }^{6}$

Decimal visual acuity data were converted into logarithm of the minimum angle of resolution ( $\log$ MAR) values, and a change of 0.0.2 logMAR was judged as improvement or worsening of vision. Hand motion was converted into a decimal visual acuity of 0.002 .

Intraoperative and postoperative complications were defined as complications that occurred during first-time PPV. Visual acuity and corneal endothelial cell density were measured for three months or longer postoperatively in cases that required second or third surgical interventions due to intraoperative or postoperative complications. Transient ocular hypertension was defined as a pressure over $25 \mathrm{mmHg}$, while transient ocular hypotension was defined as a pressure under $5 \mathrm{mmHg}$. "Transient" was used to mean a period of time that could range from a few days to 2 weeks at most.

Parameters examined in this study included the cause of dislocation, scleral fixation of the IOL, preoperative and postoperative visual acuity, preoperative and postoperative corneal endothelial cell density, and intraoperative and postoperative complications.

\section{Statistical analysis}

A paired $t$-test was used to compare preoperative and postoperative corneal endothelial cell densities. $P$ values less than 0.05 were considered to be statistically significant.

\section{Results}

\section{Causes of dislocation}

Lens dislocation was caused by ocular trauma in four eyes, was idiopathic in four eyes, and was as a result of coloboma, laser iridotomy for acute primary angle closure, and Marfan syndrome in one eye each.

\section{Scleral fixation of IOL}

Of the eleven eyes studied, scleral fixation of the IOL was performed in seven eyes, and in four eyes was not performed during first-time PPV. Of the four eyes in which scleral fixation of the IOL was not performed during first-time PPV, it was performed as a secondary procedure in two eyes, despite being initially planned to have been performed during first-time PPV, but retinal detachment had occurred because of intraoperative complications during the firsttime procedure. Scleral fixation of the IOL was therefore performed as a second procedure. In the remaining eye, a prior decision had been made to perform scleral fixation of the IOL as a second procedure. The other two eyes remained aphakic.

\section{Preoperative and postoperative visual acuity}

The initial decimal visual acuity ranged from hand motion to a visual acuity of 1.2, and $\log$ MAR values ranged from 3.699 to -0.079 . Final decimal visual acuity ranged from 0.04 to 1.2 , while the $\operatorname{logMAR}$ values ranged from 1.398 to -0.079 . When both the initial and final visual acuities were compared as $\log$ MAR values, visual acuity improved in two eyes, was unchanged in eight eyes, and worsened in one eye. The reason for the worsening in the one eye was due to retinal detachment, with this postoperative complication requiring the patient to undergo a third procedure. 


\section{Preoperative and postoperative corneal endothelial cell density}

Figure 1 shows preoperative and postoperative corneal endothelial cell densities. The mean preoperative corneal endothelial cell density was $2485 \pm 423$ (range 1294-3012) cells $/ \mathrm{mm}^{2}$ while the postoperative corneal endothelial cell density was $2301 \pm 576$ (range 1139-3194) cells $/ \mathrm{mm}^{2}$. No significant differences were seen between the eyes before and after the surgeries $(P=0.15$, paired $t$-test).

\section{Intraoperative and postoperative complications}

Intraoperative complications included retinal detachment and suprachoroidal hemorrhage in one eye each. Postoperative complications included transient ocular hypertension in three eyes, IOL dislocation in two eyes, and retinal detachment, transient ocular hypotension, vitreous hemorrhage, chronic ocular hypertension, iris capture, and vitreous incarceration of the anterior chamber in one eye each. Because there was some overlap between cases, we counted the total number of eyes.

Due to IOL dislocation and vitreous incarceration of the anterior chamber in one eye, a second operation was required. In one eye, retinal detachment required the patient to undergo a third procedure. Chronic ocular hypertension noted in one eye has required ongoing administration of a beta-blocker. The patient's ocular hypertension is now under control and is maintained at $21 \mathrm{mmHg}$.

\section{Discussion}

Problems affecting postoperative visual acuity are considered to be serious complications. In our eleven cases, retinal detachment after surgery was the most serious complication noted.

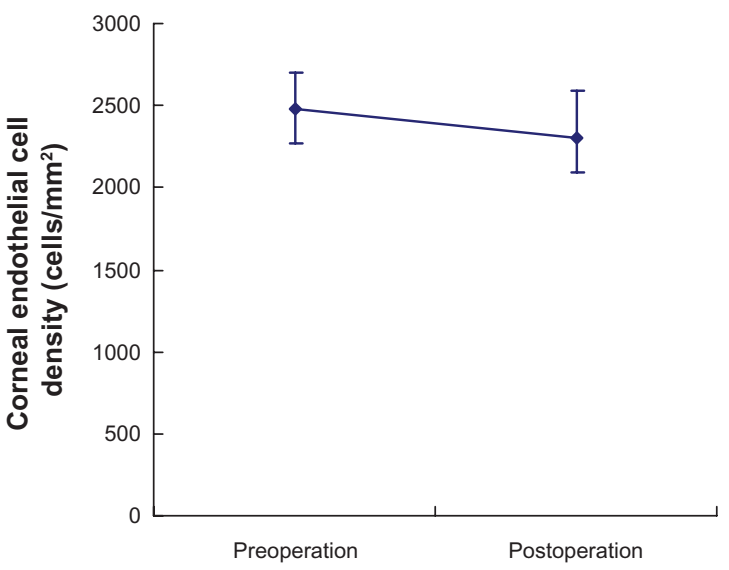

Figure I Preoperative and postoperative corneal endothelial cell density. The vertical axis represents the corneal endothelial cell density, while the horizontal axis indicates the preoperative and postoperative values.
After such surgery, the wounds need to be carefully observed. Also, vitreous strand incarceration and iatrogenic retinal tears that occur during surgery need to be correctly treated in order to avoid retinal detachment. When the procedure is properly performed, intraoperative complications, such as retinal detachments and suprachoroidal hemorrhage, can be prevented from affecting postoperative visual acuity. Postoperative ocular hypertension was reported as a complication resulting from use of perfluorocarbon liquid. ${ }^{7-9}$ Transient ocular hypertension in three eyes and chronic ocular hypertension in one eye were observed in the current study. Therefore, care should be taken when using perfluorocarbon liquid to minimize the risk of loss of postoperative visual acuity.

The most common complications in our patients resulted from ocular trauma or were idiopathic. In similar studies performed by Seo et al, ${ }^{3} \mathrm{Oh}$ and Smiddy, ${ }^{4}$ and Ogawa et al, ${ }^{10}$ it was reported that ocular trauma was the most common complication. When coloboma is present, the patient must be carefully observed because lens subluxation may be seen. ${ }^{11}$ With bullous keratopathy, although it is a known complication after laser iridotomy ${ }^{12}$ only a few cases with lens dislocation after laser iridotomy have been reported., ${ }^{3,13,14}$ Laser iridotomy can be easily performed on an outpatient basis, provided the indication for the procedure is based on a careful and thorough examination of the patient. Recently, there have been some reports supporting phacoemulsification as the first choice for acute angle closure ${ }^{15,16}$ and for angle closure glaucoma. ${ }^{16}$

The use of perfluorocarbon liquid has made it possible to remove a surgically dislocated hard lens safely. ${ }^{5}$ Because perfluorocarbon liquid has a high density, it pools posteriorly at the optic nerve, and due to its low viscosity, a smallgauge extrusion needle can be used during the procedure. Furthermore, removal of the perfluorocarbon liquid can be easily accomplished. However, when only using perfluorocarbon liquid during the procedure, it is not uncommon for lens movement to occur. Thus, the phacoemulsification technique in the vitreous cavity can be prone to difficulties when this liquid is used.

One problem that can also be encountered during the phacoemulsification procedure is small lens fragments that normally occur becoming trapped between the retina and the perfluorocarbon liquid. Removal of these fragments can be both dangerous and time-consuming. When viscoelastic is used with the perfluorocarbon liquid, these problems decrease and it is easier and safer to perform the surgery. ${ }^{1}$ Although this method was not employed in any of the eleven patients in the current study, it has now been adopted at our institution and will now be used to prevent these types of problems from occurring in the future. 
Although our PPV with the phacoemulsification method in the vitreous cavity ${ }^{17,18}$ does not require any enlargement of the scleral limbal wound, it is possible that a retinal tear or vitreous hemorrhage could occur due to the ultrasound energy present. ${ }^{19}$ Other reported methods that can be used in such cases involve lifting the lens into the anterior chamber, with removal then performed via a scleral limbal wound..$^{5,10}$ Although this method is both rapid and safe, it requires the scleral limbal wound to be enlarged. Because of this, we decided not to use this method in the current study, because it could potentially cause corectopia or iris prolapse.

We were unable to determine whether scleral fixation of an IOL during first-time PPV should be recommended in all cases. In our study, two aphakic patients elected not to undergo further surgery, so their aphakia is still present. In both cases, the patients now wear soft contact lenses for their hypermetropia. If these patients do change their minds, we will perform scleral fixation of the IOL procedure in the future.

To the best of our knowledge, there have been no previously reported studies measuring preoperative and postoperative corneal endothelial cell density. Thus, the current data will help to refine and improve the success of this technique in the future. Our results indicate that preoperative and postoperative corneal endothelial cell densities were not affected by our surgical technique. Therefore, when an experienced surgeon performs this type of surgery, the technique can be considered to be safe and one that does not cause any harm to the corneal endothelium.

To prevent inflammation or secondary glaucoma, ${ }^{2}$ it is necessary to remove a lens that has undergone complete posterior dislocation. However, in some cases, retinal detachment can occur after surgery, which could affect the visual prognosis. These complications can occur due to insufficient cutting of the vitreous body or insufficient repair of the iatrogenic retinal tear during the surgical procedure. Further studies in larger numbers of patients will need to be undertaken in the future in order to clarify the nature of these complications.

\section{Disclosure}

The authors report no conflicts of interest in this work.

Clinical Ophthalmology

\section{Publish your work in this journal}

Clinical Ophthalmology is an international, peer-reviewed journal covering all subspecialties within ophthalmology. Key topics include: Optometry; Visual science; Pharmacology and drug therapy in eye diseases; Basic Sciences; Primary and Secondary eye care; Patient Safety and Quality of Care Improvements. This journal is indexed on Submit your manuscript here: http://www.dovepress.com/clinical-ophthalmology-journal

\section{References}

1. Dalma-Weiszhausz J, Franco-Cardenas V, Dalma A. A modified technique for extracting a dislocated lens with perfluorocarbon liquids and viscoelastics. Ophthalmic Surg Lasers Imaging. 2010;41:572-574.

2. Huang HM, Kao ML, Kuo HK, et al. Visual results and complications after trans pars plana vitrectomy and lensectomy for lens dislocation. Chang Gung Med J. 2004;27:429-435.

3. Seo MS, Yoon KC, Lee CH. Phacofragmentation for the treatment of a completely posterior dislocation of the total crystalline lens. Korean J Ophthalmol. 2002;16:32-36.

4. Oh J, Smiddy WE. Pars plana lensectomy combined with pars plana vitrectomy for dislocated cataract. J Cataract Refract Surg. 2010;36:1189-1194.

5. Shapiro MJ, Resnick KI, Kim SH, et al. Management of the dislocated crystalline lens with a perfluorocarbon liquid. Am J Ophthalmol. 1991;112:401-405.

6. Mutoh T, Matsumoto Y, Chikuda M. Scleral fixation of foldable acrylic intraocular lenses in aphakic post-vitrectomy eyes. Clin Ophthalmol. 2010;5:17-21.

7. Yoshida K, Kiryu J, Kita M, et al. Phacoemulsification of dislocated lens and suture fixation of intraocular lens using a perfluorocarbon liquid. Jpn J Ophthalmol. 1998;42:471-475.

8. Sirimaharaj M, Balachandran C, Chan WC, et al. Vitrectomy with short term postoperative tamponade using perfluorocarbon liquid for giant retinal tears. Br J Ophthalmol. 2005;89:1176-1179.

9. Stolba U, Binder S, Velikay M, et al. Use of perfluorocarbon liquids in proliferative vitreoretinopathy: results and complications. Br J Ophthalmol. 1995;79:1106-1110.

10. Ogawa R, Yoshizawa T, Baba E, et al. Surgical management and visual outcome for dislocated lenses. Japanese Review of Clinical Ophthalmology. 2005;99: 551-553.

11. Jardón J, Izquierdo NJ. Coloboma and CHARGE association in Puerto Rico. Bol Asoc Med P R. 2009;101:39-41. Spanish.

12. Ang LP, Higashihara H, Sotozono C, et al. Argon laser iridotomy-induced bullous keratopathy a growing problem in Japan. Br J Ophthalmol. 2007;91:1613-1615.

13. Melamed S, Barraquer E, Epstein DL. Neodymium: YAG laser iridotomy as a possible contribution to lens dislocation. Ann Ophthalmol. 1986;18:281-282.

14. Seong M, Kim MJ, Tchah H. Argon laser iridotomy as a possible cause of anterior dislocation of a crystalline lens. J Cataract Refract Surg. 2009;35:190-192.

15. Mine M, Okamoto M, Yukawa E, et al. The criteria of surgery for acute primary angle closure in Nara Medical University. Folia Japonica de Ophthalmologica Clinica. 2010;3:1163-1166.

16. Lachkar Y. Acute angle closure and angle closure glaucoma: phacoemulsification as first-line treatment. $J$ Fr Ophtalmol. 2010;33: 273-278. French.

17. Ruiz-Moreno JM, Barile S, Montero JA. Phacoemulsification in the vitreous cavity for retained nuclear lens fragments. Eur J Ophthalmol. 2006; 16:40-45.

18. Soliman Mahdy M, Eid MZ, Shalaby KA, et al. Intravitreal phacoemulsification with pars plana vitrectomy for management of posteriorly dislocated nucleus or lens fragments. Eur J Ophthalmol. 2010;20: 115-119.

19. Bopp S, el-Hifnawi ES, Bornfeld N, et al. Retinal lesions after transvitreal use of ultrasound. Fortschr Ophthalmol. 1991;88:442-445. [German.]

\section{Dovepress}

PubMed Central and CAS, and is the official journal of The Society of Clinical Ophthalmology (SCO). The manuscript management system is completely online and includes a very quick and fair peer-review system, which is all easy to use. Visit http://www.dovepress.com/ testimonials.php to read real quotes from published authors. 